\title{
Mangiferin inhibits cell migration and angiogenesis via PI3K/AKT/mTOR signaling in high glucose- and hypoxia-induced RRCECs
}

\author{
JIA SHI $^{1}$, HONGBIN LV ${ }^{2}$, CHEN TANG ${ }^{1}$, YUJIE LI ${ }^{1}$, JING HUANG $^{1}$ and HONG ZHANG ${ }^{1}$ \\ ${ }^{1}$ Department of Ophthalmology, The People's Hospital of Deyang City, Deyang, Sichuan 618000; ${ }^{2}$ Department of \\ Ophthalmology, Affiliated Hospital of Southwest Medical University, Luzhou, Sichuan 646000, P.R. China
}

Received June 30, 2020; Accepted February 2, 2021

DOI: $10.3892 / \mathrm{mmr} .2021 .12112$

\begin{abstract}
Mangiferin is a prominent active component that can be derived from several traditional herbs, including Mangifera indica L., Anemarrhena asphodeloides Bge., and Belamcanda chinensis (L.) DC., which displays antidiabetic properties. Diabetic retinopathy (DR), a serious complication caused by diabetes, is the leading cause of blindness. The present study aimed to evaluate the beneficial effects of mangiferin on high glucose (HG)/hypoxia-induced rat retinal capillary endothelial cell (RRCEC) angiogenesis, as well as the underlying mechanisms. To establish an in vitro model of DR, RRCECs were exposed to $30 \mathrm{mM}$ glucose and hypoxia. Following treatment with different doses of mangiferin $(0.05$, 0.1 or $0.2 \mu \mathrm{M})$, RRCEC viability, migration and angiogenesis were assessed by performing Cell Counting Kit 8, immunofluorescence, wound healing, Transwell and tube formation assays. Western blotting was conducted to evaluate protein expression levels. Furthermore, LY294002 and IGF-1, an inhibitor and activator of the PI3K/AKT/mTOR signaling pathway, respectively, were used to verify the potential mechanisms underlying mangiferin. The results demonstrated that mangiferin notably inhibited HG/hypoxia-induced RRCEC migration and angiogenesis. HG/hypoxia-induced upregulation of hypoxia-inducible factor- $1 \alpha$, vascular endothelial growth factor, matrix metallopeptidase (MMP)2 and MMP9 expression levels and the phosphorylation of PI3K, AKT and mTOR in RRCECs was significantly reversed following treatment with mangiferin. Additionally, further activation of the PI3K/AKT signaling pathway by IGF-1 inhibited the beneficial effects of mangiferin on RRCECs, whereas deactivation of
\end{abstract}

Correspondence to: Professor Hongbin Lv, Department of Ophthalmology, Affiliated Hospital of Southwest Medical University, 173 Taishan North Road, Luzhou, Sichuan 646000, P.R. China E-mail: lvhongbinbiology@163.com

Key words: mangiferin, PI3K/AKT/mTOR signaling pathway, angiogenesis, diabetic retinopathy the PI3K/AKT signaling pathway by LY294002 displayed the opposite results. Collectively, the results of the present study suggested that mangiferin suppressed RRCEC angiogenesis via modulating the PI3K/AKT/mTOR signaling pathway, which could serve as an effective treatment strategy for DR.

\section{Introduction}

Diabetic retinopathy (DR) is a common complication of diabetes (1). With the increasing prevalence of diabetes, it is estimated that the number of patients with DR will reach 191 million worldwide by 2030 (2). In diabetes, hyperglycemia-mediated injury of blood vessels and capillaries causes retina ischemia and hypoxia, and vitreous hemorrhage, which leads to proliferative diabetic retinopathy, and ultimately blindness (1). At present, the local therapeutic interventions for the treatment of DR include intravitreal injection of anti-vascular endothelial growth factor (VEGF) drugs, glucocorticoids and laser photocoagulation (3). However, due to the complicated pathogenesis of DR, the current therapeutic interventions cannot completely prevent complications, including vitreous hemorrhage, traction retinal detachment and neovascular glaucoma (4). The primary pathological features of DR are retinal vascular occlusive circulation disorder, as well as retinal endothelial cell proliferation, migration and angiogenesis $(5,6)$. Therefore, inhibiting retinal neovascularization is important for the treatment of DR.

Mangiferin (1,3,6,7-tetrahydroxyxanthone-C2- $\beta$-Dglucoside) is a xanthone glucoside. Mangiferin can be extracted from plants belonging to the Liliaceae, Anacardiaceae and Gentianaceae families $(7,8)$. Mangiferin exerts a wide range of pharmacological effects, including antitumor, anti-infectious, hypoglycemic, antioxidation, and immune regulatory effects (9). A previous study demonstrated that mangiferin attenuates dexamethasone-induced osteoblastic MC3T3-E1 cell apoptosis and oxidative stress via activating the bone morphogenetic protein 2/Smad-1 signaling pathway (10). In atherosclerosis, mangiferin promoted macrophage cholesterol efflux via enhancing ATP binding cassette subfamily (ABC)A1 and ABCG1 expression (8). Moreover, it has been reported that the PI3K/AKT and Rac1/Wiskott-Aldrich syndrome protein family member 2 (WAVE2) signaling pathways are blocked 
by mangiferin in cancer (11). In particular, mangiferin exerted beneficial effects on biochemical and hematological parameters in rats with streptozotocin-induced diabetes (12). However, whether mangiferin protects against high glucose (HG)- and hypoxia-induced injury in rat retinal capillary endothelial cells (RRCECs) is not completely understood. Thus, the present study aimed to investigate the effects of mangiferin on RRCEC viability, migration and angiogenesis following exposure to $\mathrm{HG}$ and hypoxia.

\section{Materials and methods}

Drug. Mangiferin $\left(\mathrm{C}_{19} \mathrm{H}_{18} \mathrm{O}_{11}\right.$; molecular weight, 422.34; purity, $\geq 98 \%$; cat. no. 4773-96-0) was purchased from Sigma-Aldrich (Merck KGaA).

Cell culture. RRCECs (Procell Life Science \& Technology Co., Ltd.) were cultured in HG DMEM (cat. no. PM150220; Procell Life Science \& Technology Co., Ltd.) supplemented with 10\% FBS (cat. no. FB15015; Clark Bioscience) and 1\% penicillin/streptomycin (cat. no. sv30010; HyClone; Cytiva) with $5 \% \mathrm{CO}_{2}$ at $37^{\circ} \mathrm{C}$.

Cell treatment. To select the optimum concentration (A0), RRCECs were treated with different concentrations of mangiferin $(0.01,0.1,1$ or $10 \mathrm{mM})$ for 24,48 and $72 \mathrm{~h}$, as previously described $(10,13)$. Subsequently, RRCECs were pretreated with D-(+)-glucose (30 mM; cat. no. G7528; Sigma-Aldrich; Merck KGaA)/hypoxia $\left(1 \% \mathrm{O}_{2}, 94 \% \mathrm{~N}_{2}\right.$ and $\left.5 \% \mathrm{CO}_{2}\right)$ and then were treated with $0.1 \mathrm{mM}$ (A0) mangiferin for 24 or $48 \mathrm{~h}$. RRCECs were also treated with D-(+)-glucose/hypoxia and followed by different doses of mangiferin $[0.05 \mathrm{mM}$ (1/2 A0), $0.1 \mathrm{mM}$ (A0) and $0.2 \mathrm{mM}(2 \mathrm{~A} 0)]$ for $24 \mathrm{~h}$ at $37^{\circ} \mathrm{C}$. Finally, RRCECs were treated with a PI3K/AKT signaling pathway inhibitor (LY294002; Sigma-Aldrich; Merck KGaA $40 \mu \mathrm{mol} / \mathrm{l}$ ) or a PI3K/AKT signaling pathway activator (IGF-1; Sigma-Aldrich; Merck KGaA $100 \mathrm{ng} / \mathrm{ml})$ in the presence of $\mathrm{D}$-(+)-glucose/hypoxia and mangiferin (A0) for $24 \mathrm{~h}$ at $37^{\circ} \mathrm{C}$. The control cells were treated the same amount of DMSO for $24 \mathrm{~h}$ at $37^{\circ} \mathrm{C}$. Cells were divided into the following nine groups: i) Control; ii) HG/hypoxia; iii) HG/hypoxia + 1/2 A0 mangiferin; iv) $\mathrm{HG} /$ hypoxia + A0 mangiferin; v) HG/hypoxia + 2A0 mangiferin; vi) HG/hypoxia + $0.1 \mathrm{mM}$ (A0) mangiferin + LY294002; vii) and $\mathrm{HG} /$ hypoxia + 0.1 mM (A0) mangiferin + IGF-1; viii) HG/hypoxia + LY294002; ix) HG/hypoxia + IGF-1.

Western blotting. RRCECs were plated $\left(1 \times 10^{6}\right.$ cells) into $100-\mathrm{mm}$ culture plates. The following day, cells were treated with mangiferin as aforementioned. Following washing three times with PBS, total protein was extracted from cells using RIPA buffer (cat. no. P0013C; Beyotime Institute of Biotechnology) supplemented with $1 \%$ phenylmethylsulfonyl fluoride (Beyotime Institute of Biotechnology). Protein concentrations were determined using a BCA assay kit (CoWin Biosciences). Proteins (40 $\mu \mathrm{g})$ were separated alongside molecular weight standards (Bio-Rad Laboratories, Inc.) via 10\% SDS-PAGE. Proteins were transferred onto PVDF membranes (Amersham; Cytiva). Following blocking with $5 \%$ non-fat milk at room temperature for $1 \mathrm{~h}$, the membranes were incubated overnight at $37^{\circ} \mathrm{C}$ with anti-matrix metallopeptidase (MMP)2 (1:1,000; cat. no. AF5330;
Affinity Biosciences), anti-MMP9 (1:500; cat. no. ab119906; Abcam), anti-VEGF (1:1,000; cat. no. ab46154; Abcam), anti-hypoxia-inducible factor-1 $\alpha$ (HIF-1 $\alpha$; 1:500; cat. no. ab1; Abcam), anti-PI3K (1:1,000; cat. no. ab32089; Abcam), anti-AKT (1:10,000; cat. no. ab179463; Abcam), anti-mTOR (1:1,000; cat. no. ab32028; Abcam), anti-phosphorylated (p)-PI3K (1:500; cat. no. ab182651; Abcam), anti-p-AKT (1:5,000; cat. no. ab81283; Abcam), anti-p-mTOR (1:1,000; cat. no. ab109268; Abcam) and anti- $\beta$-actin (1:1,000; cat. no. AC026; ABclonal Biotech Co., Ltd.). Subsequently, the membranes were incubated with a HRP-conjugated Goat Anti-Rabbit IgG secondary antibody (1:2,000; cat. no. ab6721; Abcam) or a HRP-conjugated Goat Anti-Mouse IgG secondary antibody (1:2,000; cat. no. ab6728; Abcam) at room temperature for $3 \mathrm{~h}$. Protein bands were visualized using an ECL system (cat. no. KF001; Affinity Biosciences). Protein expression levels were semi-quantified using Image-Pro Plus software (version 6.0; Media Cybernetics, Inc.) with $\beta$-actin as the loading control.

Cell viability assay. RRCEC viability was assessed by performing Cell Counting Kit-8 (CCK-8) assays (cat. no. BS350B; Biosharp Life Sciences) according to the manufacturer's protocol. CCK-8 solution was added to each well and incubated at $37^{\circ} \mathrm{C}$ for $1.5 \mathrm{~h}$. Cell viability was measured at a wavelength of $450 \mathrm{~nm}$ using a microplate reader.

Immunofluorescence. RRCECs were seeded ( $5 \times 10^{4}$ cells/well) into 96 -well plates and cultured at $37^{\circ} \mathrm{C}$ with $5 \% \mathrm{CO}_{2}$. At $90 \%$ confluence, the culture medium was removed and cells were washed with PBS. The cells were fixed with $4 \%$ paraformaldehyde at room temperature for $30 \mathrm{~min}$ and washed 3 times with $0.02 \mathrm{M}$ PBS at room temperature for $3 \mathrm{~min}$. Following blocking with $10 \%$ serum blocking solution (cat. no. 22012-8612; Zhejiang Tianhang Biotechnology Co., Ltd.) at room temperature for $30 \mathrm{~min}$, cells were incubated with an anti-Ki67 primary antibody (1:200; cat. no. GB13030-2; Wuhan Servicebio Technology Co., Ltd.) at $4^{\circ} \mathrm{C}$ overnight. Subsequently, cells were incubated with rhodamine-labeled goat anti-rabbit IgG antibody (1:100; cat. no. ZF-0316; OriGene Technologies, Inc.) at $37^{\circ} \mathrm{C}$ for $30 \mathrm{~min}$. Finally, cell nuclei were labeled with DAPI (cat. no. ZLI-9557; OriGene Technologies, Inc.) at room temperature for $10 \mathrm{~min}$ and the cells were incubated with anti-fluorescence decay blocking solution at room temperature (cat. no. AR1109; Wuhan Boster Biological Technology, Ltd.). Stained cells were observed using a fluorescence microscope (magnification, x400; Olympus Corporation).

Tube formation assay. First, $60 \mu \mathrm{l}$ dissolved Matrigel adhesive matrix was added into each well of a pre-cooled 96-well plate and allowed to solidify at $37^{\circ} \mathrm{C}$ for $1 \mathrm{~h}$. RRCECs $\left(5 \times 10^{4}\right.$ cells $\left./ \mathrm{ml}\right)$ were added into each well, cultured under hypoxic conditions $\left(1 \% \mathrm{O}_{2}, 94 \% \mathrm{~N}_{2}\right.$ and $\left.5 \% \mathrm{CO}_{2}\right)$ for $6 \mathrm{~h}$ and then transferred to normoxic conditions (95\% air and $5 \% \mathrm{CO}_{2}$ ) for $12 \mathrm{~h}$. Subsequently, cells were treated with mangiferin $(0.05,0.1$ or $0.2 \mathrm{mM}$ ) at $37^{\circ} \mathrm{C}$ with $5 \% \mathrm{CO}_{2}$ for $24 \mathrm{~h}$. Tube formation was observed under an inverted phase-contrast light microscope in five randomly selected fields of view.

Wound healing assay. RRCEC migration was assessed by performing a wound healing assay. Briefly, cells were grown 

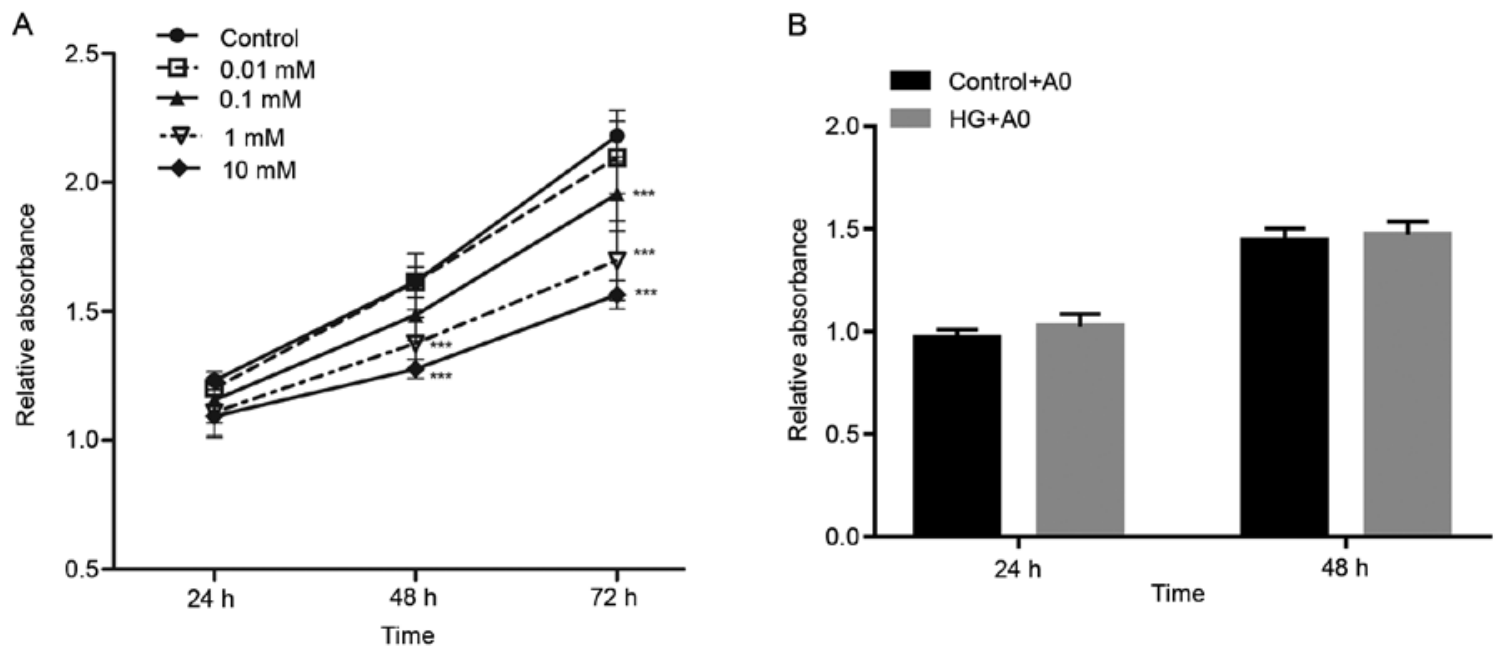

Figure 1. Effects of mangiferin on HG/hypoxia-induced RRCEC viability. RRCECs were treated with different doses of mangiferin $(0.01,0.1,1 \mathrm{and} 10 \mathrm{mM})$ for 24,48 or $72 \mathrm{~h}$. (A and B) Cell viability was assessed by performing Cell Counting Kit- 8 assays. ${ }^{* * * *} \mathrm{P}<0.001$ vs. control. HG, high glucose; RRCEC, rat retinal capillary endothelial cell; A0, $0.1 \mathrm{mM}$ mangiferin.

in DMEM medium containing 10\% FBS and then seeded $\left(5 \times 10^{5}\right.$ cells) into a 6 -well plate. At $100 \%$ confluence, a wound was created in each cell monolayer using a sterile micropipette tip, followed by washing with PBS to remove cell debris. Subsequently, cells were cultured in serum-free DMEM under hypoxic conditions for $6 \mathrm{~h}$, and then transferred to normoxic conditions for $12 \mathrm{~h}$. Finally, cells were treated with different concentrations of mangiferin $(0.05,0.1$ or $0.2 \mathrm{mM})$ for $24 \mathrm{~h}$ at $37^{\circ} \mathrm{C}$ with $5 \% \mathrm{CO}_{2}$. An inverted phase-contrast light microscope (Olympus Corporation) was used to obtain images of the wound at different time points. The width of the wound was measured at three different positions at 0 and $24 \mathrm{~h}$. The relative wound recovery distance was calculated as $0-24 \mathrm{~h}$.

Transwell assay. To evaluate cell migration, RRCECs were resuspended $\left(1 \times 10^{6} \mathrm{cells} / \mathrm{ml}\right)$ in serum-free DMEM. Transwell chambers were placed into a 24 -well plate. Subsequently, cell suspension (20 $\mu \mathrm{l} /$ well) was added into the upper chamber. Cells were cultured under hypoxic conditions for $6 \mathrm{~h}$ and then normoxic conditions for $12 \mathrm{~h}$. Subsequently, $600 \mu \mathrm{l}$ DMEM supplemented with 10\% FBS (cat. no. FB15015; Clark Bioscience) was added into the lower chamber. Cells in the upper chamber were treated with mangiferin $(0.05$, 0.1 or $0.2 \mathrm{mM}$ ) for $24 \mathrm{~h}$ and cultured at $37^{\circ} \mathrm{C}$ with $5 \% \mathrm{CO}_{2}$. Following fixation with $4 \%$ paraformaldehyde for $20 \mathrm{~min}$ at room temperature, cells were stained with $0.1 \%$ crystal violet for $15 \mathrm{~min}$ at room temperature. Migratory cells were visualized using an inverted phase-contrast light microscope (Olympus Corporation).

Statistical analysis. Statistical analysis was performed on the means of triplicates that resulted from three independent replications of each experiment. Data are presented as the mean \pm SD. Statistical analyses were performed using SPSS software (version 20.0; IBM Corp.). Comparisons among groups were analyzed using one-way ANOVA followed by Tukey's post hoc test. $\mathrm{P}<0.05$ was considered to indicate a statistically significant difference.

\section{Results}

Effect of mangiferin on HG/hypoxia-induced RRCEC viability. Cell viability was assessed by performing CCK- 8 assays. The results demonstrated that mangiferin $(0.01-10 \mathrm{mM})$ decreased RRCEC viability in a dose-dependent manner (Fig. 1). Furthermore, treatment of RRCECs with mangiferin for 24,48 and $72 \mathrm{~h}$ increased cell viability in a time-dependent manner (Fig. 1). Following treatment with A0 mangiferin for $48 \mathrm{~h}$, no significant alteration in cell viability was observed compared with the control group (Fig. 1A). In addition, RRCECs were treated with $\mathrm{A} 0$ mangiferin and induced with $\mathrm{HG}$ /hypoxia for 24 and $48 \mathrm{~h}$. Co-treatment of RRCECs with HG and A0 mangiferin for 24 and $48 \mathrm{~h}$ did not significantly alter cell viability compared with the control + A0 group (Fig. 1B). Therefore, the results indicated that $\mathrm{A} 0$ mangiferin did not display cytotoxicity.

Mangiferin attenuates HG/hypoxia-induced RRCEC migration and angiogenesis. Subsequently, the effects of mangiferin on HG/hypoxia-induced viability, migration and angiogenesis were investigated. Compared with the control group, HG/hypoxia exposure significantly increased RRCEC viability, whereas mangiferin did not display significant inhibitory or promotive effects on RRCEC viability (Fig. 2A). Furthermore, the immunofluorescence results demonstrated that compared with the control group, the number of $\mathrm{Ki}^{6} 7^{+}$cells was clearly increased following treatment with HG/hypoxia, an effect that was not notably reversed by mangiferin (Fig. 2B). In addition, HG/hypoxia exposure significantly increased RRCEC migration compared with the control group (Fig. 2C-F). Treatment with $1 / 2 \mathrm{~A} 0$, A0 or $2 \mathrm{~A} 0$ mangiferin markedly attenuated HG/hypoxia-mediated effects on cell migration (Fig. 2C-F). Compared with the control group, RRCEC angiogenesis was markedly enhanced following treatment with HG/hypoxia, which was notably attenuated by treatment with $1 / 2 \mathrm{~A} 0, \mathrm{~A} 0$ or $2 \mathrm{~A} 0$ mangiferin (Fig. 2G). Furthermore, following exposure to HG/hypoxia, the expression levels of HIF-1 $\alpha$, VEGF, MMP2 and MMP9 in RRCECs were significantly upregulated compared with the control group. However, treatment with 
A

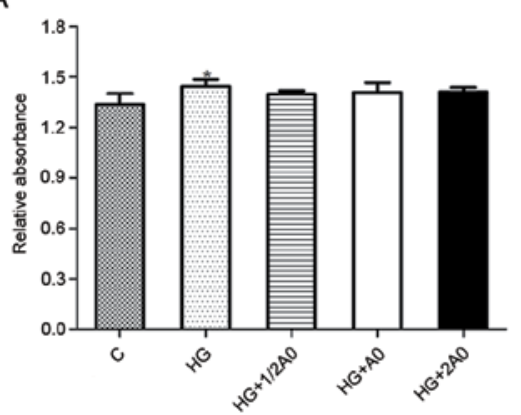

B

c

HG

$H G+1 / 2 A O$

HG+AO

$H G+2 A O$
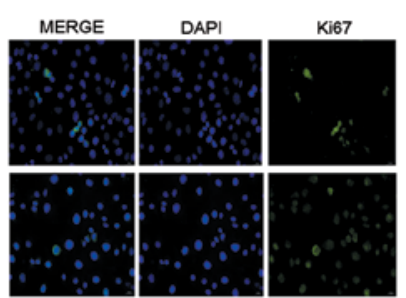

F
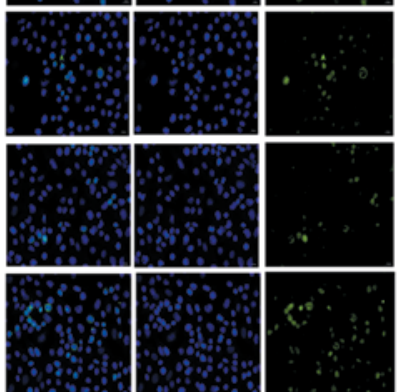

$\mathrm{H}$
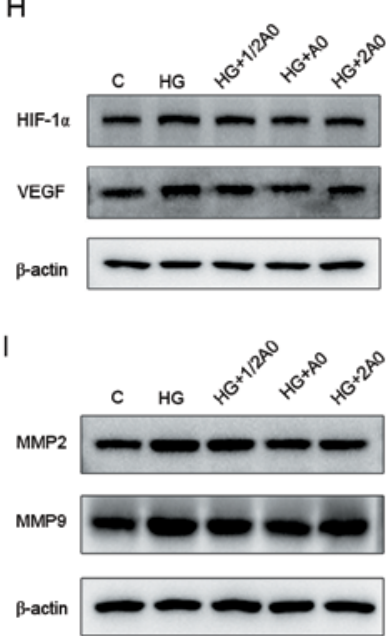

G
C
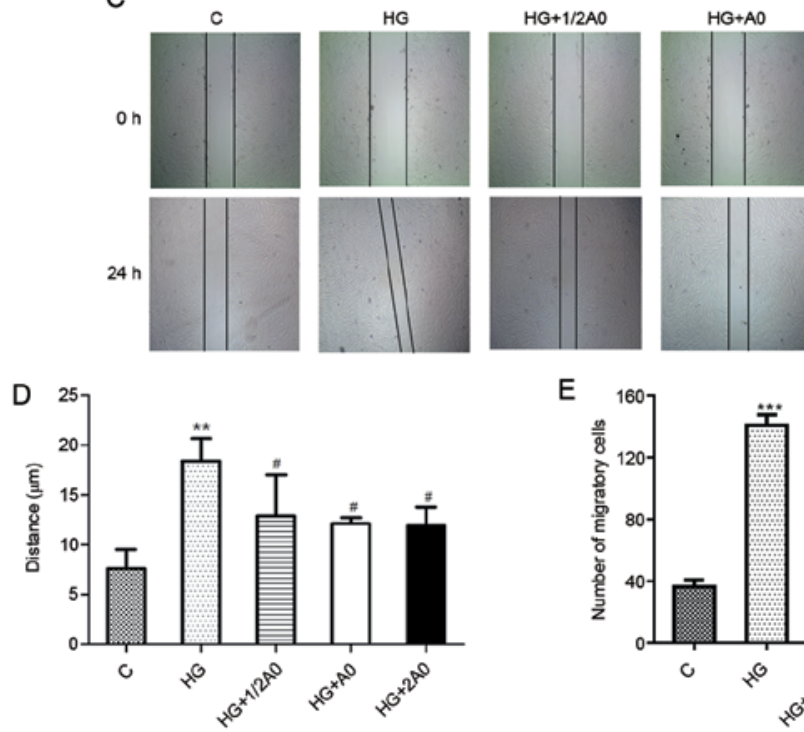

E

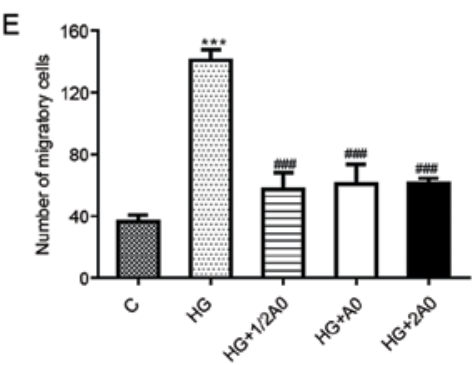

C
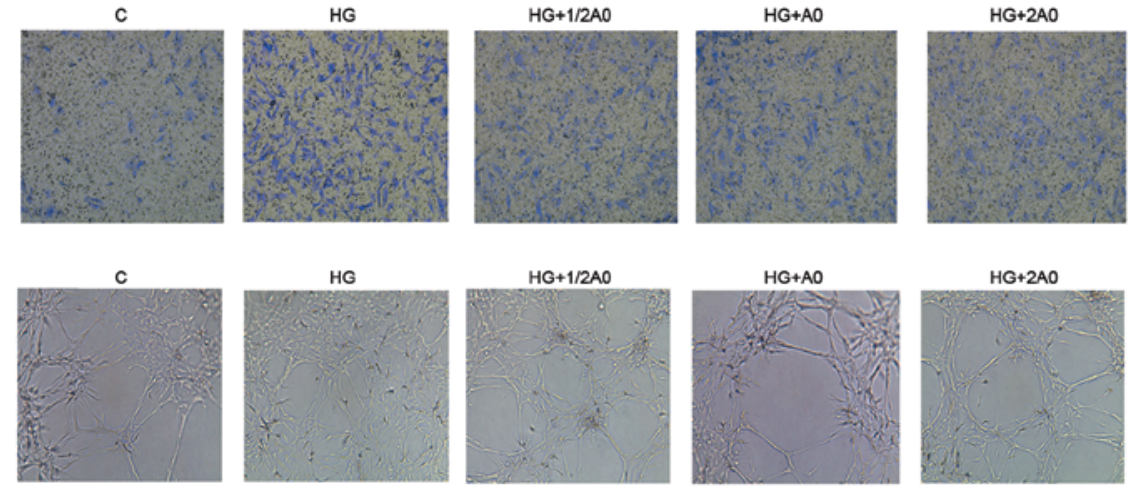

$H G+1 / 2 A O$

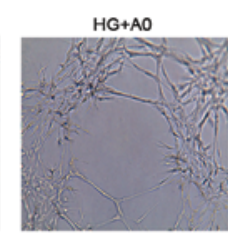

$\mathrm{HG}+2 \mathrm{AO}$
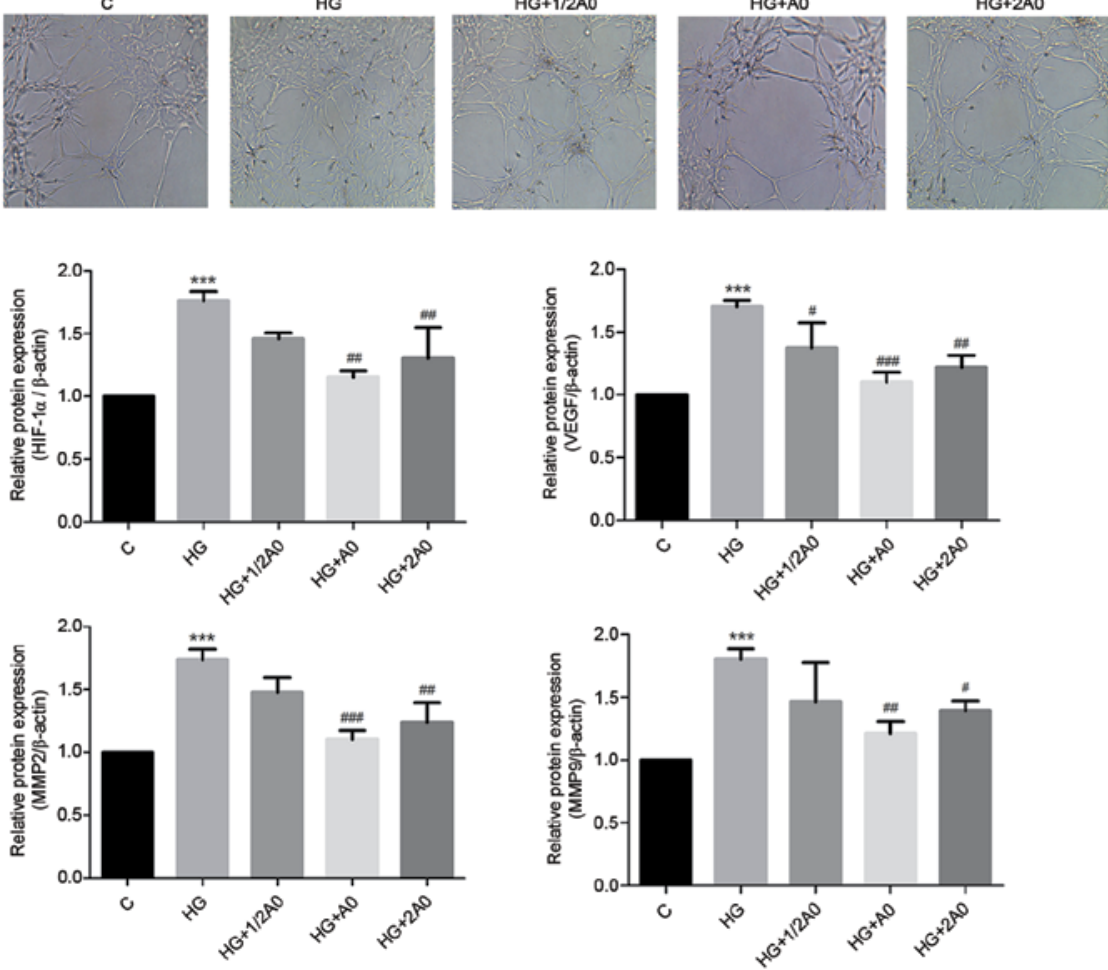

Figure 2. Mangiferin attenuates HG/hypoxia-induced RRCEC migration and angiogenesis. (A) RRCEC viability was evaluated by performing Cell Counting Kit-8 assays. (B) Ki67 expression was determined via immunofluorescence (magnification, $\mathrm{x} 400)$. RRCEC migration was (C) determined by performing wound healing assay (magnification, x50) and (D) quantified. (E) Quantification of RRCEC migration as determined by (F) performing Transwell assays (magnification, x100). (G) Tube formation and branching points were determined (magnification, x100). Protein expression levels of (H) HIF-1 $\alpha$, VEGF, (I) MMP2 and MMP9 were determined via western blotting. ${ }^{* *} \mathrm{P}<0.01$ and ${ }^{* * *} \mathrm{P}<0.001$ vs. control; ${ }^{\#} \mathrm{P}<0.05,{ }^{\# \#} \mathrm{P}<0.01$ and ${ }^{\# \# \#} \mathrm{P}<0.001$ vs. HG. HG, high glucose; RRCEC, rat retinal capillary endothelial cell; HIF-1 $\alpha$, hypoxia-inducible factor-1 $\alpha$; VEGF, vascular endothelial growth factor; MMP2, matrix metallopeptidase 2; C, control; 1/2 A0, $0.05 \mathrm{mM}$ mangiferin; A0, 0.1 mM mangiferin; 2A0, $2 \mathrm{mM}$ mangiferin.

A0 or $2 \mathrm{~A} 0$ mangiferin significantly reversed $\mathrm{HG} /$ hypoxia exposure-induced upregulation of HIF-1 $\alpha$, VEGF, MMP2 and MMP9 expression levels (Fig. 2H and I).
Mangiferin suppresses the activity of the PI3K/AKT and mTOR signaling pathways in HG/hypoxia-treated RRCECs. The PI3K/AKT and mTOR signaling pathways serve important 


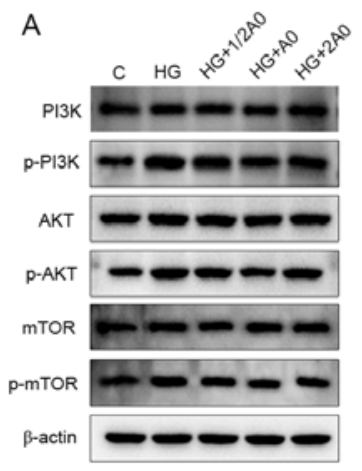

B

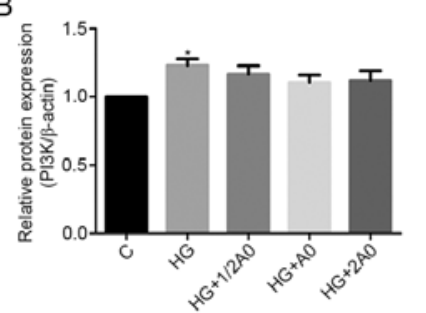

$\mathrm{E}$

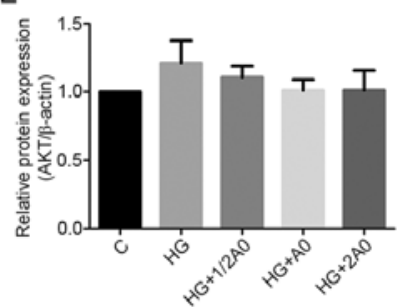

$\mathrm{H}$

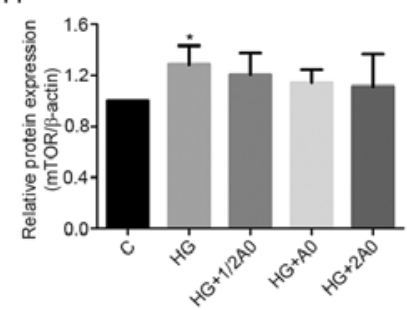

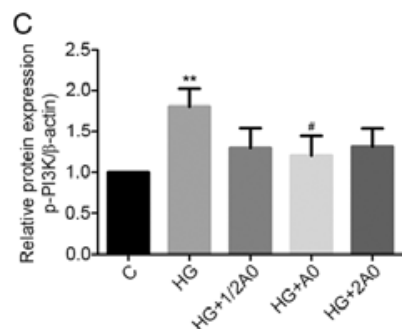

F

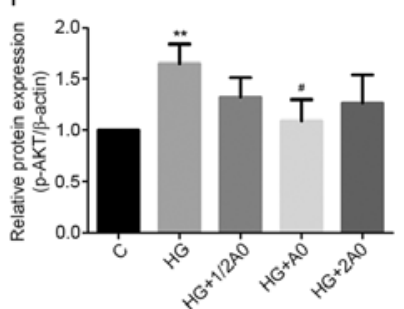

I

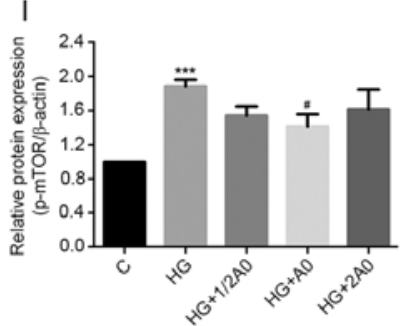

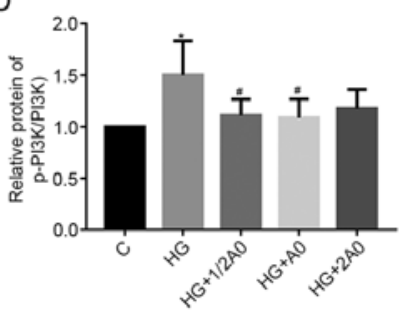

G
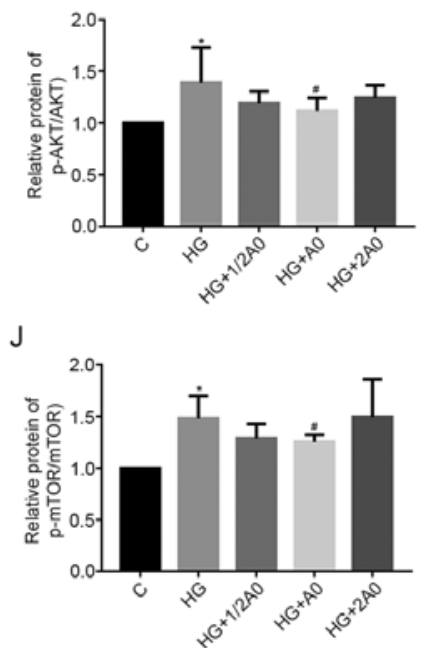

Figure 3. Mangiferin suppresses PI3K/AKT/mTOR signaling pathway activation in HG/hypoxia-treated RRCECs. Protein expression levels were (A) determined via western blotting and semi-quantified for (B PI3K, (C) p-PI3K, (D) p-PI3K/PI3K, (E) AKT, (F) p-AKT, (G) p-AKT/AKT, (H) mTOR, (I) p-mTOR and (J) p-mTOR/mTOR. ${ }^{*} \mathrm{P}<0.05,{ }^{* *} \mathrm{P}<0.01$ and ${ }^{* * *} \mathrm{P}<0.001$ vs. control; ${ }^{*} \mathrm{P}<0.05$ vs. HG. HG, high glucose; RRCEC, rat retinal capillary endothelial cell; p, phosphorylated; C, control; 1/2 A0, $0.05 \mathrm{mM}$ mangiferin; A0, $0.1 \mathrm{mM}$ mangiferin; 2A0, $2 \mathrm{mM}$ mangiferin.

roles in various intracellular events, including cell migration, invasion and angiogenesis $(14,15)$. Therefore, the present study explored whether these signaling pathways were implicated in mangiferin-mediated cell migration and angiogenesis. The western blotting results demonstrated that the protein expression levels of PI3K, p-PI3K, p-AKT, mTOR and p-mTOR were significantly upregulated in $\mathrm{HG} /$ hypoxia-exposed cells compared with control cells (Fig. 3). However, the levels of PI3K, AKT and mTOR phosphorylation were significantly decreased following treatment with A0 mangiferin in HG/hypoxia-exposed cells (Fig. 3). Similar results were observed for the p-AKT/AKT, p-PI3K/PI3K and p-mTOR/mTOR ratios (Fig. 3D, G and J).

Mangiferin inhibits HG/hypoxia-induced RRCEC migration and angiogenesis via the PI3K/AKT and mTOR signaling pathways. The present study investigated whether the PI3K/AKT and mTOR signaling pathways were involved in cell migration and angiogenesis following exposure to HG/hypoxia. The western blotting results demonstrated that the PI3K/AKT signaling pathway inhibitor (LY294002) and the PI3K/AKT signaling pathway activator (IGF-1) significantly downregulated and upregulated the expression levels of p-PI3K, p-AKT and p-mTOR in HG/hypoxia-exposed and mangiferin-treated RRCECs, respectively (Fig. 4A-J). Mangiferin-mediated downregulation of p-AKT/AKT, p-PI3K/PI3K and p-mTOR/mTOR ratios was significantly reversed by IGF-1 and significantly enhanced by LY294002 (Fig. 4A-J). However, RRCEC viability was not significantly altered among the groups (Fig. 4K).
Additionally, mangiferin-induced inhibition of RRCEC migration was significantly enhanced and inhibited following treatment with LY294002 and IGF-1, respectively (Fig. 4L-O). Furthermore, the tube formation assay results demonstrated that A0 mangiferin markedly reduced the number of branching points in HG/hypoxia-treated cells, an effect that was notably enhanced and inhibited following treatment with LY294002 and IGF-1, respectively (Fig. 4P). Overall, the results of the present study suggested that mangiferin inhibited RRCEC migration and angiogenesis partially via deactivation of the PI3K/AKT and mTOR signaling pathways.

\section{Discussion}

Long-term exposure to hyperglycemia and hypoxia is highly associated with the development of DR pathological states, and is considered the primary cause of blood-retinal barrier and microvascular injury (16). Mangiferin, a natural bioactive ingredient, is commonly found in mango trees, and has been reported to display antitumor, antidiabetic, lipometabolism regulating, anti-inflammatory and antibacterial properties (17). Previous studies have reported that mangiferin exerts pharmacological effects on hypoglycemia and diabetes. In streptozotocin-induced type 2 diabetic rats, mangiferin ameliorates insulin sensitivity and dyslipidemia, depletes serum TNF- $\alpha$ and elevates adiponectin (18). Mangiferin suppresses increased plasma insulin and fasted plasma non-esterified fatty acid concentrations at the baseline during an oral glucose tolerance test in a rat model of fructose-induced metabolic 

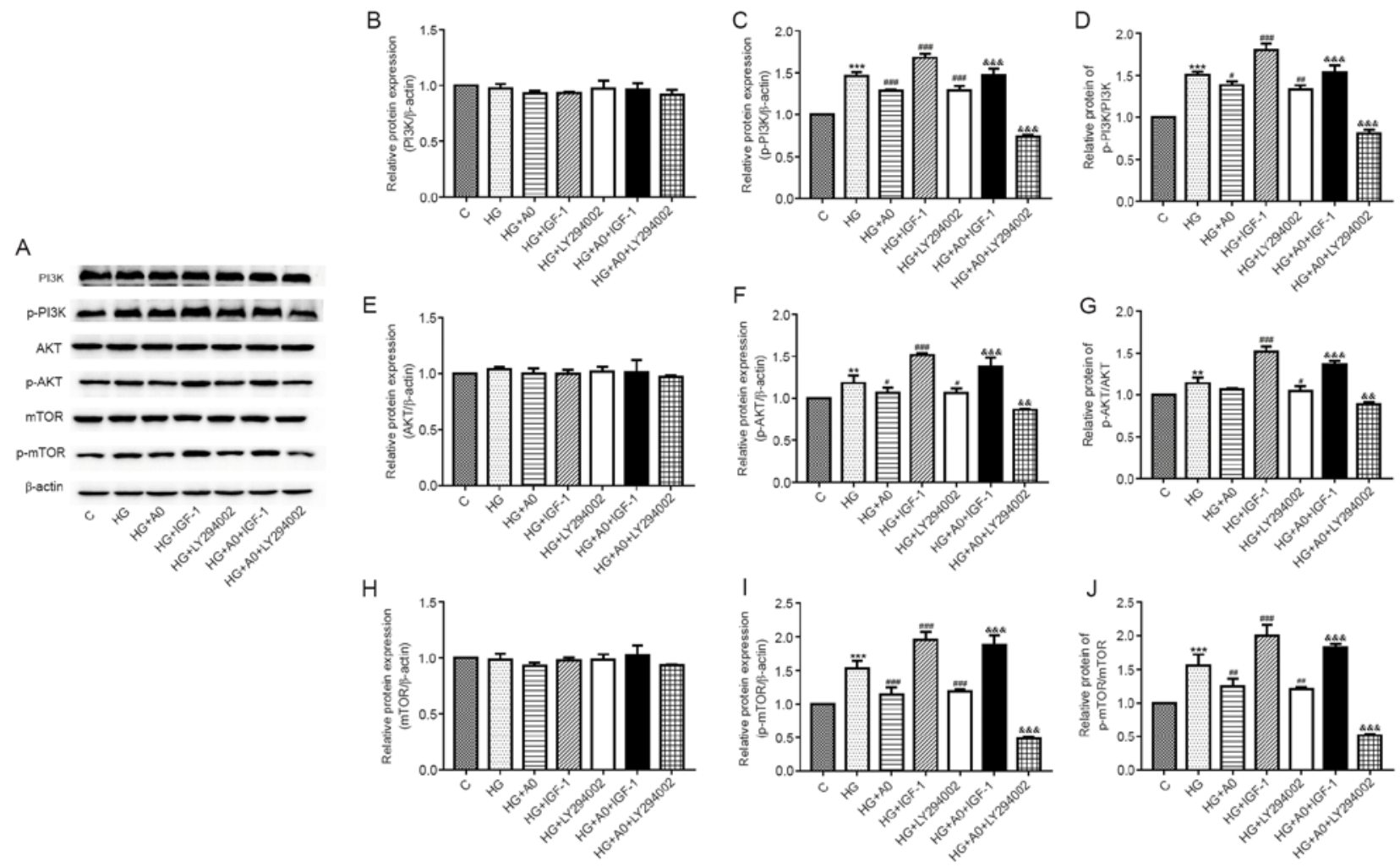

K

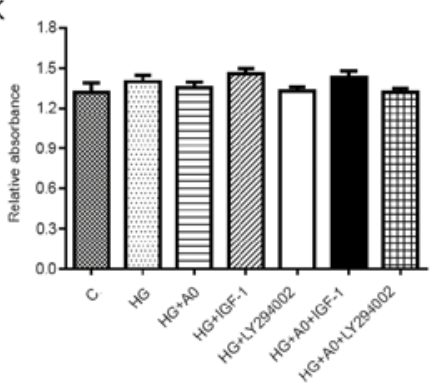

N
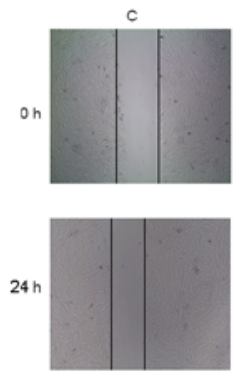

o

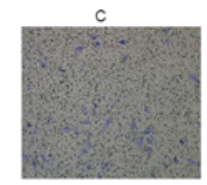

P
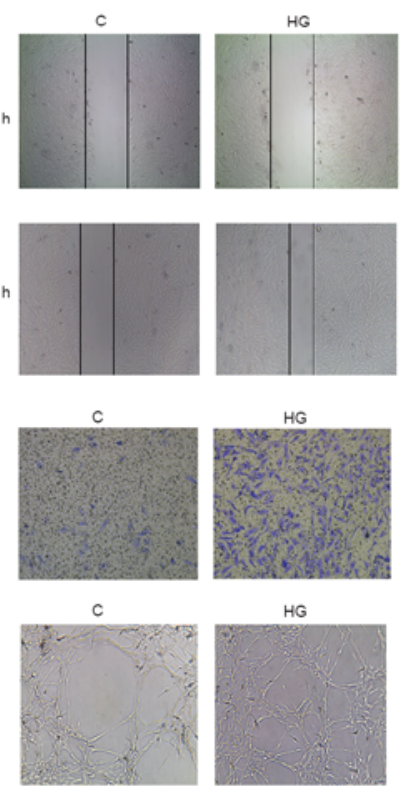

HG

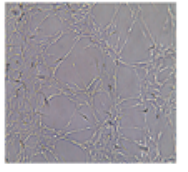

L

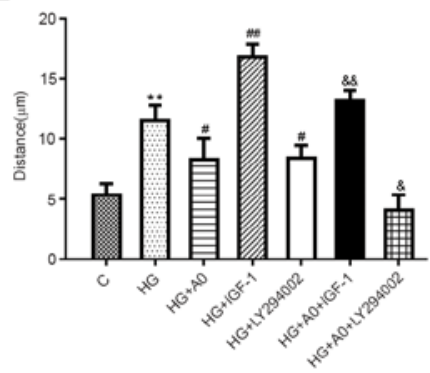

M
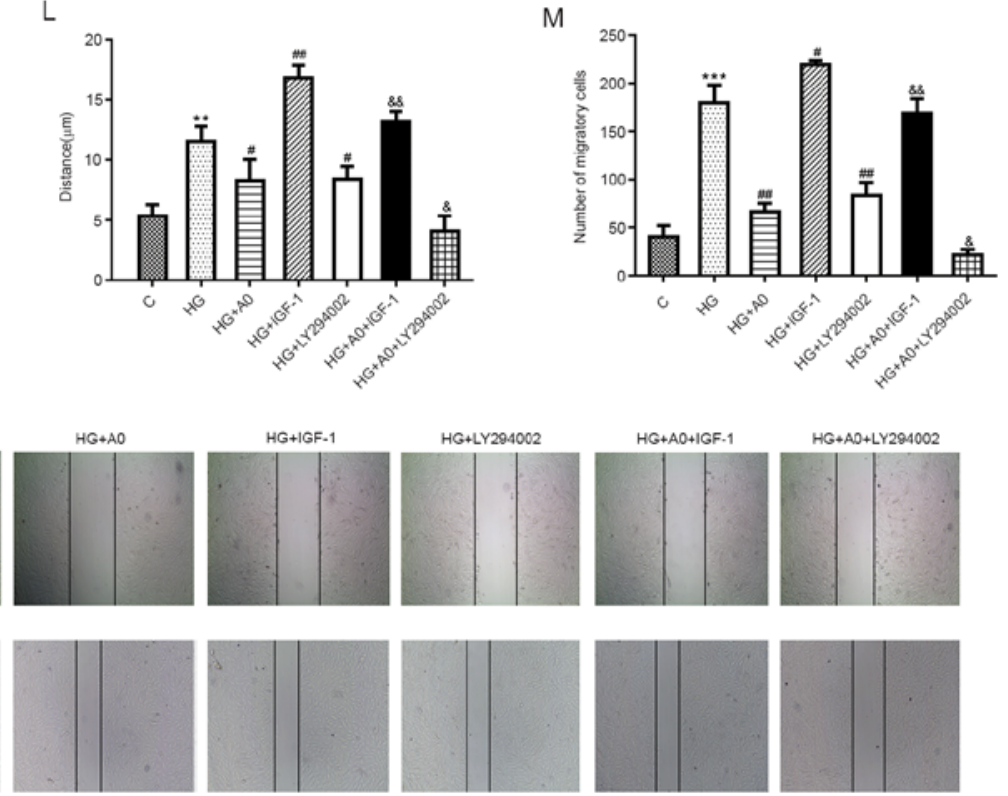

HG+AO
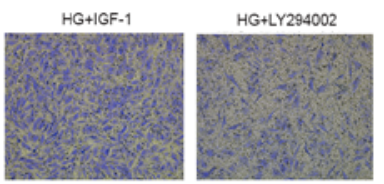

HG+AO+1GF-1

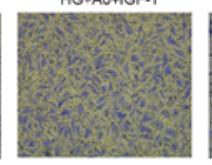

HG+A0+LY294002

HG+IGF-1

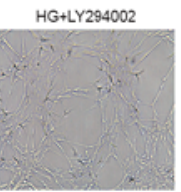

HG+A0+1FG-1
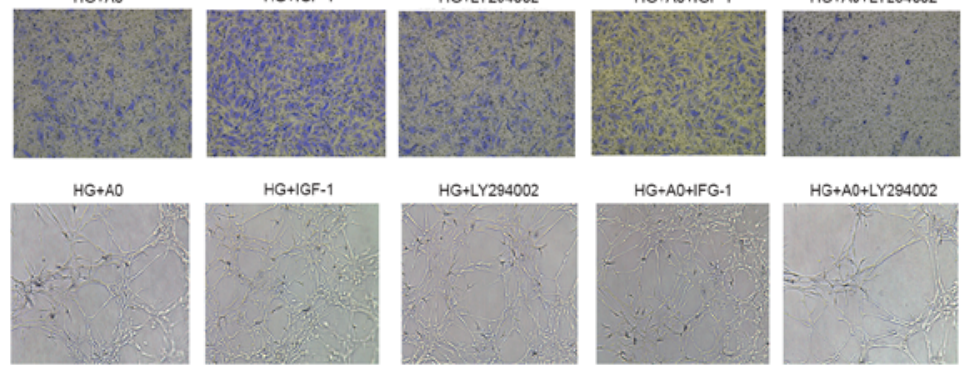

HG+A0+LY 294002

Figure 4. Mangiferin inhibits HG/hypoxia-induced cell migration and angiogenesis via the PI3K/AKT/mTOR signaling pathways in RRCECs. Protein expression levels were (A) determined via western blotting and semi-quantified for (B) PI3K, (C) p-PI3K, (D) p-PI3K/PI3K, (E) AKT, (F) p-AKT, (G) p-AKT/ AKT, (H) mTOR, (I) p-mTOR and (J) p-mTOR/mTOR. (K) RRCEC viability was evaluated by performing Cell Counting Kit-8 assays. RRCEC migration was evaluated by performing (L) wound healing and (M) Transwell assay. Representative images of the (N) wound healing (x50) and (O) Transwell assays (magnification, $\mathrm{x} 100)$. ( $\mathrm{P})$ Tube formation and branching points were determined magnification, $\mathrm{x} 100) .{ }^{* *} \mathrm{P}<0.01$ and ${ }^{* * * *} \mathrm{P}<0.001$ vs. control; ${ }^{\#} \mathrm{P}<0.05,{ }^{\# \#} \mathrm{P}<0.01$ and ${ }^{\# \# \#} \mathrm{P}<0.001$ vs. HG; ${ }^{\&} \mathrm{P}<0.05,{ }^{\& \&} \mathrm{P}<0.01$ and ${ }^{\& \& \&} \mathrm{P}<0.001$ vs. HG+A0. HG, high glucose; RRCEC, rat retinal capillary endothelial cell; p, phosphorylated; $\mathrm{C}$, control; A0, $0.1 \mathrm{mM}$ mangiferin. 
syndrome (19). Furthermore, at 3 weeks post-oral administration, mangiferin decreases blood glucose levels in KK-Ay mice, but displays no effect on blood glucose levels in healthy mice (20). Moreover, mangiferin clearly improves reactive oxygen species (ROS) generation, inflammation and endoplasmic reticulum stress in gestational diabetes mellitus model mice (21). Mangiferin increases bovine aortic cell migration, but displays no effect on cell proliferation in vitro (22). The results of the present study demonstrated that mangiferin displayed no significant effect on cell viability in HG/hypoxia-induced RRCECs. Additionally, treatment with mangiferin markedly attenuated HG/hypoxia-induced RRCEC migration and angiogenesis, which suggested that mangiferin may be useful for the treatment of DR. Mechanistically, mangiferin inhibits glioma cell proliferation and promotes apoptosis via downregulating MMP9 expression (23). Furthermore, mangiferin reduces MMP7 and MMP9 expression, and inhibits breast cancer cell proliferation and epithelial-mesenchymal transition (EMT) in vitro and in vivo via activation of the $\beta$-catenin signaling pathway (24). Increasing evidence has demonstrated that mangiferin attenuates inflammation via inhibiting the Toll-like receptor 4/p65 signaling pathway, and suppresses the EMT process via blocking the Smad2/3 signaling pathway and downregulating MMP9 expression in pulmonary fibrosis model mice (25). Similarly, the results of the present study demonstrated that mangiferin significantly decreased MMP2 and MMP9 expression levels in HG/hypoxia-exposed RRCECs.

HIF- $\alpha$ /VEGF expression serves a major role in mediating active retinal angiogenesis and alleviating the progression of DR. In DR, HIF- $1 \alpha$ regulates the transcription of VEGF, which ultimately leads to intracellular accumulation of VEGF (26). VEGF is an important stimulating factor involved in retinal and choroidal neovascularization $(27,28)$. Clinical data indicates that the concentration of VEGF is enhanced in vitreous fluid of patients with DR (29). It has been reported that anti-VEGF agents may be used to treat angiogenesis in eye diseases, including neovascular glaucoma, persistent vitreous hemorrhage and DR (30). In the present study, mangiferin treatment significantly downregulated HG/hypoxia-induced HIF- $\alpha$ and VEGF expression levels.

In addition, several signaling pathways, including the PI3K/AKT, STAT3 and mTOR signaling pathways, are associated with HIF-1 $\alpha /$ VEGF signaling in DR (31-33). It has been reported that the PI3K/AKT/mTOR signaling pathway promotes DR via inducing retinal cell viability, apoptosis, migration and angiogenesis. The use of a combination of PI3K/AKT/mTOR drugs is safe and effective for the treatment of ocular neovascularization (34). In retinal endothelial RF/6A and microglia BV-2 cells, erianin significantly suppresses $\mathrm{HG}$-induced increases in retinal vessels and microglia activation via deactivating the VEGF/PI3K/AKT signaling pathway (35). Furthermore, microRNA-183 overexpression activates the PI3K/AKT signaling pathway, increases VEGF expression and downregulates B-cell translocation gene 1 expression in DR (36). In addition, Nogo-B induced VEGF secretion and activation of the PI3K/AKT axis in HG-exposes human retinal microvascular endothelial cells (37). A previous study reported that Parkinsonism associated deglycase overexpression significantly reduces
ROS production, $\mathrm{p}-\mathrm{AKT}$ and $\mathrm{p}$-mTOR expression, and cell apoptosis in HG-induced retinal capillary pericytes (38). Similarly, the present study demonstrated that A0 mangiferin significantly inhibited the PI3K/AKT/mTOR signaling pathway in HG/hypoxia-induced RRCECs. Activation of the PI3K/AKT signaling pathway by IGF-1 clearly reversed mangiferin-mediated effects on RRCEC migration and angiogenesis. Moreover, A20 mangiferin did not significantly reduce the phosphorylation levels of PI3K, AKT and mTOR in HG/hypoxia-induced RRCECs, which suggested that high concentration mangiferin was not conducive to the inhibition of protein phosphorylation. It was hypothesized that high concentrations of mangiferin might activate or inhibit additional upstream signaling pathways to impair the phosphorylation levels of PI3K, AKT and mTOR; however, further investigations are required to confirm the effects of high concentration mangiferin on the upstream components of the PI3K/AKT signaling pathway.

In summary, to the best of our knowledge, the present study demonstrated for the first time that mangiferin displayed beneficial effects against DR by suppressing HG/hypoxia-induced RRCEC migration and angiogenesis via inhibition of the $\mathrm{PI} 3 \mathrm{~K} / \mathrm{AKT} / \mathrm{mTOR}$ signaling pathway, suggesting the potential use of mangiferin for the treatment of DR.

\section{Acknowledgements}

Not applicable.

\section{Funding}

This study was supported by the Sichuan Province Science and Technology Support Program (grant no. 2015SZ0086).

\section{Availability of data and materials}

The datasets used or analyzed during the current study are available from the corresponding author on reasonable request.

\section{Authors' contributions}

JS, CT, YL and HL conceptualized and designed the study. JS, CT, YL, JH and HZ performed the experiments. JS, CT and YL analyzed the data. JS, CT, YL and HL drafted the manuscript. JS and HL confirmed the authenticity of all the raw data. All authors read and approved the final version of the manuscript.

\section{Ethics approval and consent to participate}

Not applicable.

\section{Patient consent for publication}

Not applicable.

\section{Competing interests}

The authors declare that they have no competing interests. 


\section{References}

1. Cheung N, Mitchell P and Wong TY: Diabetic retinopathy. Lancet 376: 124-136, 2010.

2. Ting DS, Cheung GC and Wong TY: Diabetic retinopathy: global prevalence, major risk factors, screening practices and public health challenges: a review. Clin Exp Ophthalmol 44: 260-277, 2016.

3. Wang W and Lo AC: Diabetic retinopathy: Pathophysiology and treatments. Int J Mol Sci 19: 1816, 2018.

4. Whitehead M, Wickremasinghe S, Osborne A, Van Wijngaarden P and Martin KR: Diabetic retinopathy: A complex pathophysiology requiring novel therapeutic strategies. Expert Opin Biol Ther 18: $1257-1270,2018$

5. Kim D, Lee D, Trackman PC and Roy S: Effects of high glucose-induced lysyl oxidase propeptide on retinal endothelial cell survival: Implications for diabetic retinopathy. Am J Pathol 189 1945-1952, 2019.

6. Mei X,Zhou L,Zhang T,Lu B, Sheng Y and Ji L: Chlorogenic acid attenuates diabetic retinopathy by reducing VEGF expression and inhibiting VEGF-mediated retinal neoangiogenesis. Vascul Pharmacol 101: 29-37, 2018.

7. Imran M, Arshad MS, Butt MS, Kwon JH, Arshad MU and Sultan MT: Mangiferin: A natural miracle bioactive compound against lifestyle related disorders. Lipids Health Dis 16: 84, 2017

8. Ren K, Li H, Zhou HF, Liang Y, Tong M, Chen L, Zheng XL and Zhao GJ: Mangiferin promotes macrophage cholesterol efflux and protects against atherosclerosis by augmenting the expression of ABCA1 and ABCG1. Aging (Albany NY) 11: 10992-11009, 2019.

9. Núñez Selles AJ, Daglia M and Rastrelli L: The potential role of mangiferin in cancer treatment through its immunomodulatory, anti-angiogenic, apoptopic, and gene regulatory effects. Biofactors 42: 475-491, 2016

10. Ding LZ, Teng X, Zhang ZB, Zheng CJ and Chen SH: Mangiferin inhibits apoptosis and oxidative stress via BMP2/Smad-1 signaling in dexamethasone-induced MC3T3-E1 cells. Int J Mol Med 41: 2517-2526, 2018.

11. Deng Q, Tian YX and Liang J: Mangiferin inhibits cell migration and invasion through Racl/WAVE2 signalling in breast cancer Cytotechnology 70: 593-601, 2018.

12. Sellamuthu PS, Arulselvan P, Fakurazi S and Kandasamy M Beneficial effects of mangiferin isolated from Salacia chinensis on biochemical and hematological parameters in rats with streptozotocin-induced diabetes. Pak J Pharm Sci 27: 161-167, 2014

13. Zhang Q, Kong X, Yuan H, Guan H, Li Y and Niu Y: Mangiferin improved palmitate-induced-insulin resistance by promoting free fatty acid metabolism in HepG2 and $\mathrm{C} 2 \mathrm{C} 12$ cells via PPAR $\alpha$ : mangiferin improved insulin resistance. J Diabetes Res 2019 : $2052675,2019$.

14. Karar J and Maity A: PI3K/AKT/mTOR pathway in angiogenesis Front Mol Neurosci 4: 51, 2011.

15. Huang W, Ding X, Ye H, Wang J, Shao J and Huang T: Hypoxia enhances the migration and invasion of human glioblastoma U87 cells through PI3K/Akt/mTOR/HIF-1 $\alpha$ pathway. Neuroreport 29: $1578-1585,2018$

16. Nyengaard JR, Ido Y, Kilo C and Williamson JR: Interactions between hyperglycemia and hypoxia: Implications for diabetic retinopathy. Diabetes 53: 2931-2938, 2004.

17. Du S, Liu H, Lei T, Xie X, Wang H, He X, Tong R and Wang Y Mangiferin: An effective therapeutic agent against several disorders (Review). Mol Med Rep 18: 4775-4786, 2018.

18. Saleh S, El-Maraghy N, Reda E and Barakat W: Modulation of diabetes and dyslipidemia in diabetic insulin-resistant rats by mangiferin: Role of adiponectin and TNF- $\alpha$. An Acad Bras Cienc 86: 1935-1948, 2014.

19. Zhou L, Pan Y, Chonan R, Batey R, Rong X, Yamahara J, Wang J and Li Y: Mitigation of insulin resistance by mangiferin in a rat model of fructose-induced metabolic syndrome is associated with modulation of CD36 redistribution in the skeletal muscle. J Pharmacol Exp Ther 356: 74-84, 2016.

20. Miura T, Ichiki H, Hashimoto I, Iwamoto N, Kato M, Kubo M, Ishihara E, Komatsu Y, Okada M, Ishida T, et al: Antidiabetic activity of a xanthone compound, mangiferin. Phytomedicine 8 $85-87,2001$

21. Sha H, Zeng H, Zhao J and Jin H: Mangiferin ameliorates gestational diabetes mellitus-induced placental oxidative stress, inflammation and endoplasmic reticulum stress and improves fetal outcomes in mice. Eur J Pharmacol 859: 172522, 2019.
22. Daud NH, Aung CS, Hewavitharana AK, Wilkinson AS, Pierson JT, Roberts-Thomson SJ, Shaw PN, Monteith GR, Gidley MJ and Parat MO: Mango extracts and the mango component mangiferin promote endothelial cell migration. J Agric Food Chem 58: 5181-5186, 2010.

23. Xiao J, Liu L, Zhong Z, Xiao C and Zhang J: Mangiferin regulates proliferation and apoptosis in glioma cells by induction of microRNA-15b and inhibition of MMP-9 expression. Oncol Rep 33: 2815-2820, 2015.

24. Li H, Huang J, Yang B, Xiang T, Yin X, Peng W, Cheng W, Wan J, Luo F, Li H, et al: Mangiferin exerts antitumor activity in breast cancer cells by regulating matrix metalloproteinases, epithelial to mesenchymal transition, and $\beta$-catenin signaling pathway. Toxicol Appl Pharmacol 272: 180-190, 2013.

25. Jia L, Sun P, Gao H, Shen J, Gao Y, Meng C, Fu S, Yao H and Zhang G: Mangiferin attenuates bleomycin-induced pulmonary fibrosis in mice through inhibiting TLR4/p65 and TGF- $\beta 1 / \mathrm{Smad} 2 / 3$ pathway. J Pharm Pharmacol 71: 1017-1028, 2019.

26. Wang N, Zhang $\mathrm{C}$, Tan $\mathrm{H}$, et al Insulin accelerates progression of diabetic retinopathy through activating HIF-1 $\alpha /$ VEGF pathway in retinal endothelial cells. 2019.

27. Gao X, Li Y, Wang H, Li C and Ding J: Inhibition of HIF-1a decreases expression of pro-inflammatory IL- 6 and TNF- $\alpha$ in diabetic retinopathy. Acta Ophthalmol 95: e746-e750, 2017.

28. Zhang D, Lv FL and Wang GH: Effects of HIF-1 $\alpha$ on diabetic retinopathy angiogenesis and VEGF expression. Eur Rev Med Pharmacol Sci 22: 5071-5076, 2018.

29. Aiello LP, Avery RL, Arrigg PG, Keyt BA, Jampel HD, Shah ST, Pasquale LR, Thieme H, Iwamoto MA, Park JE, et al: Vascular endothelial growth factor in ocular fluid of patients with diabetic retinopathy and other retinal disorders. N Engl J Med 331: 1480-1487, 1994.

30. Osaadon P, Fagan XJ, Lifshitz T and Levy J: A review of anti-VEGF agents for proliferative diabetic retinopathy. Eye (Lond) 28: 510-520, 2014

31. Cui J, Gong R, Hu S, Cai L and Chen L: Gambogic acid ameliorates diabetes-induced proliferative retinopathy through inhibition of the HIF-1 $\alpha$ /VEGF expression via targeting PI3K/AKT pathway. Life Sci 192: 293-303, 2018

32. Yang $X$, Cao J, Du Y, Gong $Q$, Cheng $Y$ and $\mathrm{Su} \mathrm{G}$ : Angiopoietin-like protein 4 (ANGPTL4) induces retinal pigment epithelial barrier breakdown by activating signal transducer and activator of transcription 3 (STAT3): Evidence from ARPE-19 cells under hypoxic condition and diabetic rats. Med Sci Monit 25: 6742-6754, 2019.

33. Wei J, Jiang H, Gao $\mathrm{H}$ and Wang G: Blocking mammalian target of rapamycin (mTOR) attenuates HIF-1 $\alpha$ pathways engaged-vascular endothelial growth factor (VEGF) in diabetic retinopathy. Cell Physiol Biochem 40: 1570-1577, 2016.

34. Sasore T and Kennedy B: Deciphering combinations of $\mathrm{PI} 3 \mathrm{~K} / \mathrm{AKT} / \mathrm{mTOR}$ pathway drugs augmenting anti-angiogenic efficacy in vivo. PLoS One 9: e105280, 2014.

35. Yu Z, Zhang T, Gong C, Sheng Y, Lu B, Zhou L, Ji L and Wang Z: Erianin inhibits high glucose-induced retinal angiogenesis via blocking ERK1/2-regulated HIF-1 $\alpha$-VEGF/VEGFR2 signaling pathway. Sci Rep 6: 34306, 2016.

36. Zhang ZZ, Qin XH and Zhang J: MicroRNA-183 inhibition exerts suppressive effects on diabetic retinopathy by inactivating BTG1-mediated PI3K/Akt/VEGF signaling pathway. Am J Physiol Endocrinol Metab 316: E1050-E1060, 2019.

37. Zhang Y, Wang L, Zhang Y, Wang M, Sun Q, Xia F, Wang R and Liu L: Nogo-B Promotes angiogenesis in proliferative diabetic retinopathy via VEGF/PI3K/Akt pathway in an autocrine manner. Cell Physiol Biochem 43: 1742-1754, 2017.

38. Zeng J, Zhao $\mathrm{H}$ and Chen B: DJ-1/PARK7 inhibits high glucose-induced oxidative stress to prevent retinal pericyte apoptosis via the $\mathrm{PI} 3 \mathrm{~K} / \mathrm{AKT} / \mathrm{mTOR}$ signaling pathway. Exp Eye Res 189: 107830, 2019.

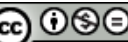

This work is licensed under a Creative Common Attribution-NonCommercial-NoDerivatives 4.0 International (CC BY-NC-ND 4.0) License. 